\title{
Sympathetic cutaneous vasomotor alerting responses (SCVARs) are associated with hippocampal theta rhythm in non-moving conscious rats
}

\author{
Rodrigo Cunha Alvim de Menezes*, Youichirou Ootsuka, William W. Blessing \\ Centre for Neuroscience, Departments of Physiology and Medicine, Flinders University, Adelaide, SA, Australia
}

\section{A R T I C L E I N F O}

Article history:

Accepted 13 August 2009

Available online 21 August 2009

Keywords:

Blood flow

Non-noxious alerting stimuli

SCVAR index

Electroencephalogram

Vasoconstriction

\begin{abstract}
A B S T R A C T
Alerting stimuli that cause sympathetic cutaneous vasomotor alerting responses ("SCVARs") in the thermoregulatory vascular bed of the rabbit ear pinna also induce theta (5-8 Hz) rhythm in the hippocampal EEG, a marker that the animal is engaged with the environment. The present study determines the relation between SCVARs in the thermoregulatory tail artery vascular bed and hippocampal EEG theta rhythm in SpragueDawley rats. A Doppler ultrasonic flow probe chronically implanted around the base of the tail artery was used to measure SCVARs. Unipolar electrodes were implanted in the hippocampus (CA1 region) to measure EEG. Six standard non-noxious brief alerting stimuli were administered during continuous recording of tail blood flow and EEG. The SCVAR index was calculated as the percentage fall from pre-alerting blood flow values. After stimuli the SCVAR index for the tail artery blood flow was $84 \pm 2 \%$ of the pre-alerting control. In contrast, the same stimuli caused little fall in blood flow within the superior mesenteric bed (SCVAR index $=18 \pm 2 \%)$. The proportion of theta power in the total frequency range $(0-20 \mathrm{~Hz})$ increased significantly after alerting stimuli $(46 \pm 2 \%$ vs. $29 \pm 1 \%$ before stimuli, $p<0.05)$. Theta proportion began to increase approximately $0.5 \mathrm{~s}$ after the stimuli and preceded SCVARs by approximately $1 \mathrm{~s}$. The SCVAR index was correlated with the magnitude of the increment in theta power. Our study demonstrates that alerting responses resulting in selective vasoconstriction of the tail vascular bed are associated with hippocampal theta rhythm in conscious rats.
\end{abstract}

(c) 2009 Elsevier B.V. All rights reserved.

\section{Introduction}

Perception of a dangerous, or potentially dangerous, environmental event triggers a complex series of defensive responses. The particular response depends on the immediacy and nature of the threat, so that the classical defense "fight or flight" dichotomy is not always appropriate. In rats, specific defensive behaviors include cognitive orientation to deter- mine the location and the nature of the possible threat, a process of risk assessment (Blanchard and Blanchard, 1988, 1989). The first phase of risk assessment includes "freezing," with scanning movements of the head and sniffing, and "stretch approach posture," with an elongated stationary body orientated towards the stimulus. When context of a cued or potential, rather than an actual, threat is presented, risk assessment is the most common behavior (Blanchard and

* Corresponding author. Neurology Laboratory, Department of Human Physiology, School of Medicine, Flinders University, Bedford Park, SA 5042, Australia. Fax: +61 882045768.

E-mail address: rodrigo.menezes@flinders.edu.au (R.C.A. de Menezes). 
Blanchard, 1988, 1989; Blanchard et al., 2001). This type of behavior may occur in conjunction with the orienting reflex, which is characterized by increased attention towards a novel stimulus. Additionally, simultaneous changes in blood flow distribution, represented by dilation of cerebral vessel and constriction of cutaneous vessels, arise during the orienting reflex (Sokolov, 1963).

Physiological components of "freezing" include sudden sympathetically mediated vasoconstriction in the thermoregulatory cutaneous vascular beds, for example, in the ear pinnae in rabbits, the tail in rats, and the fingers and toes in humans (Blessing, 2005; Blessing and Ootsuka, 2007; Kistler et al., 1998; Yu and Blessing, 1997b, 1999). Blessing (2005) introduced the term SCVARs to refer to these "sympathetic cutaneous vasomotor alerting responses." SCVARs in rabbits are accompanied by increases in blood flow to the brain, perhaps a preparatory response for increased brain processing necessary for risk assessment (Yu and Blessing, 1997a). In agreement with this proposal, SCVARs in rabbits are preceded by the appearance of theta rhythm in the hippocampal electroencephalogram (EEG) (Yu and Blessing, 1997b).

Hippocampal theta rhythm occurs in association with voluntary movement and navigation, with memory and learning, and with alerting responses (Berry and Seager, 2001; Bland, 1986; Sainsbury et al., 1987; Vanderwolf, 1969; Vertes and Kocsis, 1997; Vertes, 2005). Theta rhythm associated with voluntary movement (e.g. rearing, exploring) is referred to as type 1 theta, and usually appears in a frequency range of 8 $12 \mathrm{~Hz}$. Theta rhythm accompanying the freezing response is referred to as type 2 theta, with a frequency of 4-9 Hz (Bland, 1986). This latter form of theta is elicited by sensory stimuli in various species including rabbits and rats (Bland et al., 1981; Montoya et al., 1989; Sainsbury and Montoya, 1984; Sainsbury et al., 1987; Yu and Blessing, 1997b).

In immobile rats, hippocampal theta can be elicited with presentation of a natural predator (Sainsbury et al., 1987). In rats the occurrence of theta depends on the modality of the sensory stimulus presented, with touch and visual stimuli producing trains of theta and with neutral sound tones less effective. However in high-arousal conditions even a neutral sound can elicit theta (Montoya et al., 1989; Sainsbury et al., 1987). Thus stimulus-elicited theta may indicate that the stimulus is relevant to the animal (i.e. salient), eliciting the risk assessment process that occurs during situations of potential danger.

The present study determines whether non-noxious alerting stimuli that induce SCVARs in the rat tail artery bed also elicit hippocampal theta rhythm. The specificity of the cardiovascular effect was assessed by comparing changes in tail artery blood flow after alerting stimuli with simultaneously measured changes in blood flow to the superior mesenteric bed.

\section{Results}

\subsection{Effect of alerting stimuli on tail artery SCVARs and on mesenteric artery blood flow}

Alerting stimuli caused tail blood artery flow to fall, with the reduction in flow commencing approximately $1.4 \mathrm{~s}$ after stimulus administration (Figs. $1 \mathrm{~A}$ and 2A). The SCVAR index for all alerting stimuli was $83 \pm 2 \%$ (Fig. 3B), indicating that stimuli caused a decrease in mean tail blood flow and tail pulse amplitude to $17 \pm 2 \%$ of values measured just before the administration of the stimuli. There was no difference in the SCVAR index between stimuli $\left(F_{(1,5)}=1.3\right.$, $\mathrm{p}=0.3, n=7)$. Alerting stimuli did not produce any reproducible changes in HR regardless of the type of stimuli (data not shown).

In rats with both tail and mesenteric Doppler probes alerting stimuli caused SCVARs in the tail artery bed (SCVAR index $87 \pm 3 \%$ ) (Figs. 2A and B). In contrast, the same stimuli caused little change in blood flow in the superior mesenteric bed (Fig. 2A), so that the SCVAR index in response to alerting stimuli in this bed was $18 \pm 2 \%$, significantly less than the tail bed $(p<0.0001)$ (Fig. 2B).

\subsection{Effect of alerting stimuli on hippocampal EEG}

Alerting stimuli were administered when the animal was still and the hippocampal EEG exhibited an irregular mixed wave pattern (Figs. 1A and B). The stimuli changed the EEG pattern, causing the appearance of theta rhythm (5-8 Hz) within approximately $0.4 \mathrm{~s}$ (Fig. 1B). After this time, the proportion of $5-8 \mathrm{~Hz}$ theta power versus total power was significantly higher compared with pre-stimulus proportions (Figs. 3A and B). During the next $10 \mathrm{~s}$, the proportion of theta gradually increased $(46 \pm 2 \%$ after $10 \mathrm{~s}$ vs. $29 \pm 1 \%$ before stimuli, $\mathrm{p}<0.05)$ (Fig. 3B). After the peak, theta proportion gradually decreased returning to values similar to baseline values approximately $25 \mathrm{~s}$ after stimuli (Fig. 3B).

\subsection{Hippocampal EEG and SCVARs}

The increase in theta proportion was accompanied by a reduction in tail blood flow (Fig. 3A). The SCVAR index was correlated with the magnitude of the increment in theta proportion (significant linear regression, $F_{(1,33)}=6.2, p=0.02$, $r^{2}=0.16,34$ stimuli, $n=7$ ) (Fig. 3C). In all rats for which data were used for the final analysis, the histology confirmed that at least one EEG electrode was positioned in the CA1 area in the dorsal hippocampus (Fig. 4). After most stimuli rats tended to remain motionless, however lateral movements of the head could be observed characterizing a risk assessment behavior.

\section{Discussion}

Our results show, for the first time in rats, that acute vasoconstriction in the tail artery vascular bed produced non-noxious alerting stimuli that is associated with hippocampal theta rhythm. These results are in agreement with our previous experiments in rabbits ( $\mathrm{Yu}$ and Blessing, 1997b). In addition, we confirm our previous observations in rabbits and rats that vasoconstriction elicited by salient non-noxious alerting stimuli cause sudden substantial vasoconstriction in the thermoregulatory cutaneous vascular beds, with a much reduced effect on blood flowing to the mesenteric bed (Blessing, 2005; Blessing and Ootsuka, 2007; Yu and Blessing, 

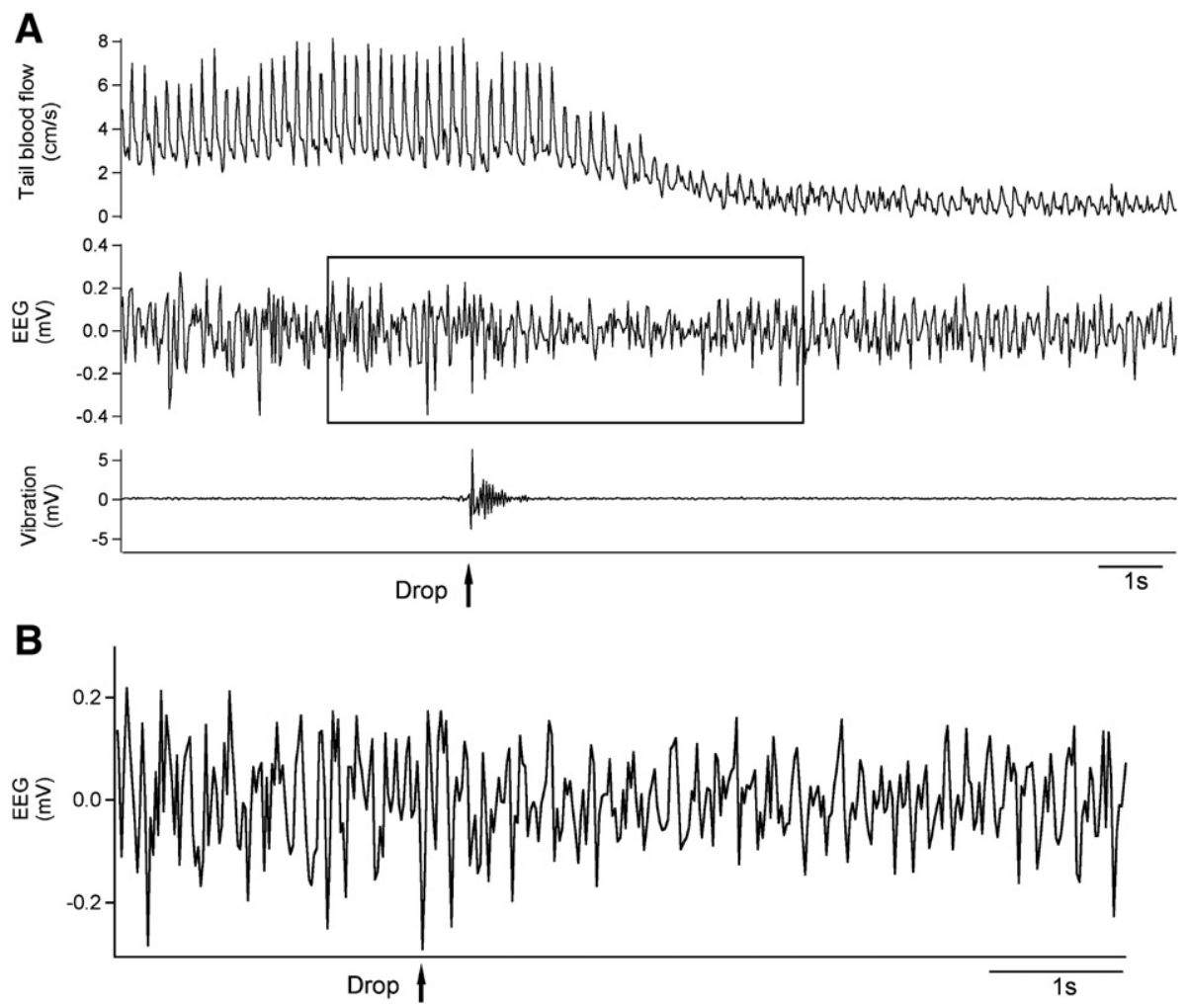

Fig. 1 - Effects of stimuli in the tail artery blood flow and in the hippocampal EEG. Tail artery pulsatile blood flow (A), hippocampal EEG (A, B), and vibration detector device signals (A). (A) Example of changes in tail artery blood flow and hippocampal EEG evoked by drop. Blood flow rapidly decreases and is preceded by a change of EEG frequency pattern (increasing theta power). (B) Magnification of the BOX in A detailing the changes in EEG caused by Drop (see text). EEG clearly changed to a more rhythmic pattern (theta) after the stimulus.

1997b). The same non-noxious stimuli had only minor effects on heart rate, with no consistent pattern of increase or decrease in this variable. Thus, sudden thermoregulatory cutaneous vasoconstriction is a vigorous and specific physiological indication that the animal has detected a salient environmental stimulus.
The vasoconstriction of the tail due to stimuli presentation depends on activation of areas in the brain that are related with defence responses including the medullary raphe region. For instance, inactivation of medullary raphe neurons in rats prevented falls in cutaneous blood flow that normally occur in response to alerting stimuli (Ootsuka and Blessing, 2005). On
A

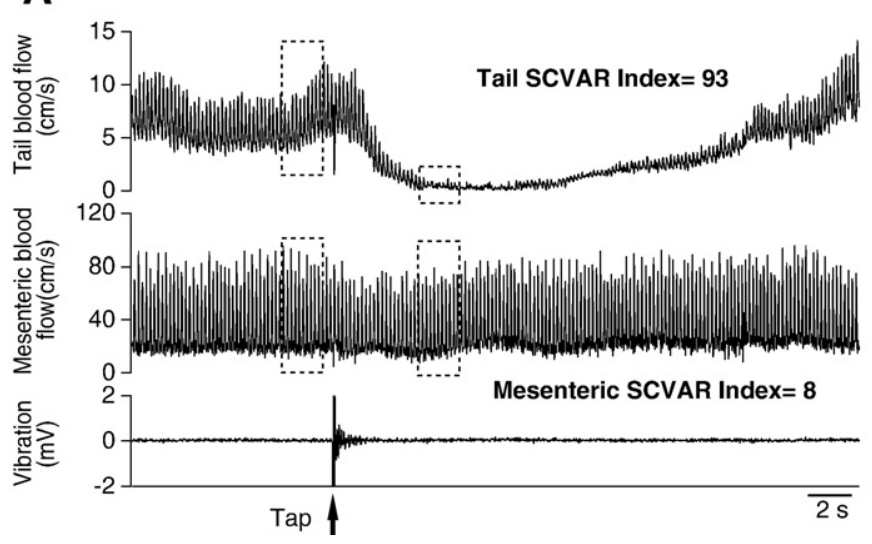

B

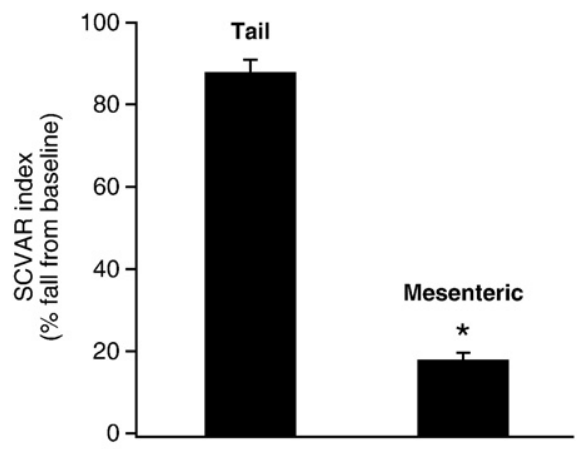

Fig. 2 - Comparison of the effect of stimuli between tail and mesenteric artery blood flow. (A) Example of changes induced by a stimulus (Tap) in tail artery blood flow and mesenteric artery blood flow. Stimulus caused SCVAR in the tail artery (sustained vasoconstriction) but not in the mesenteric artery. (B) Group data (mean \pm SEM) showing the SCVAR index produced by stimuli in both tail and mesenteric arteries blood flow. "Significantly less than tail artery SCVAR index, p $<0.0001$. 

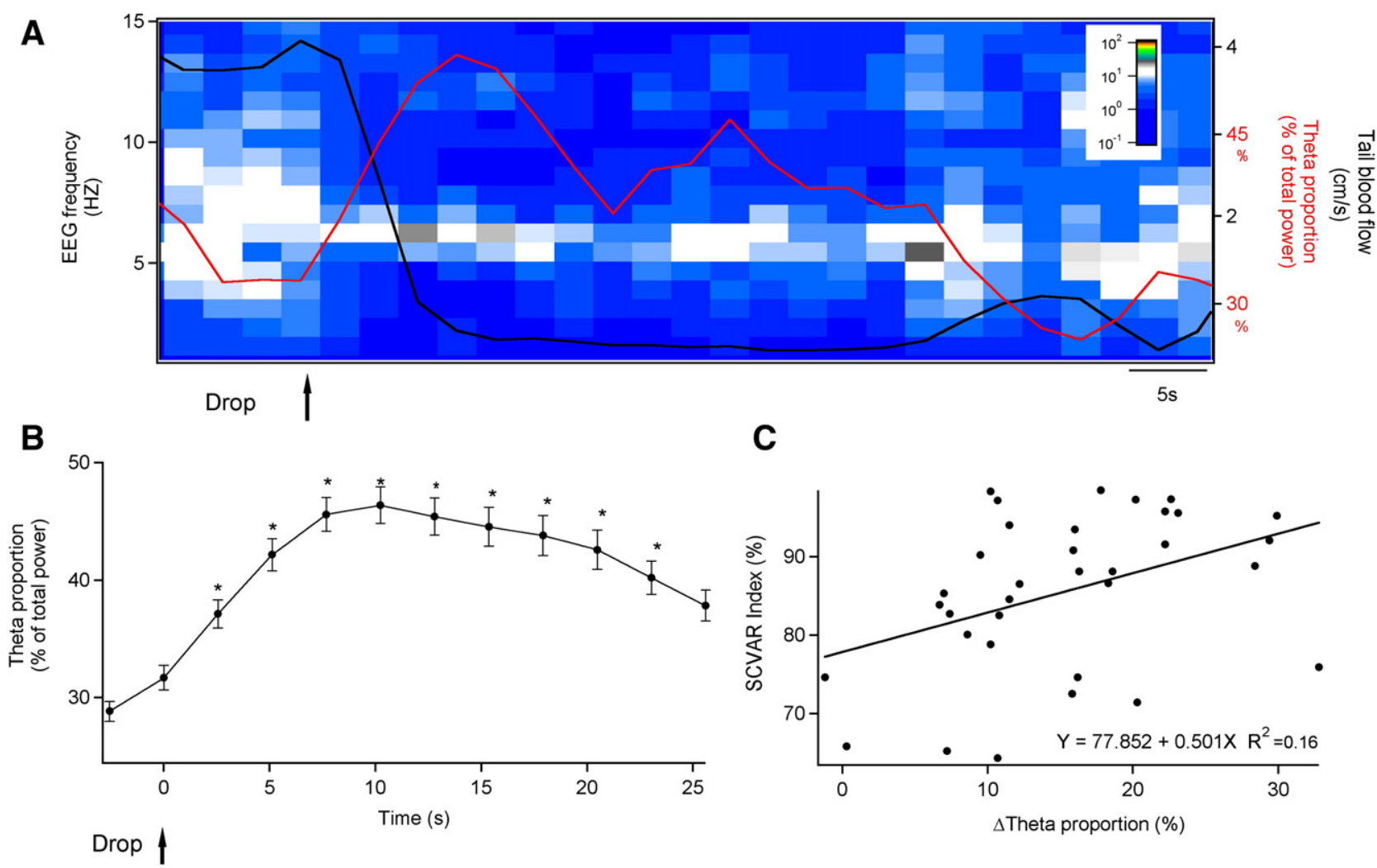

C

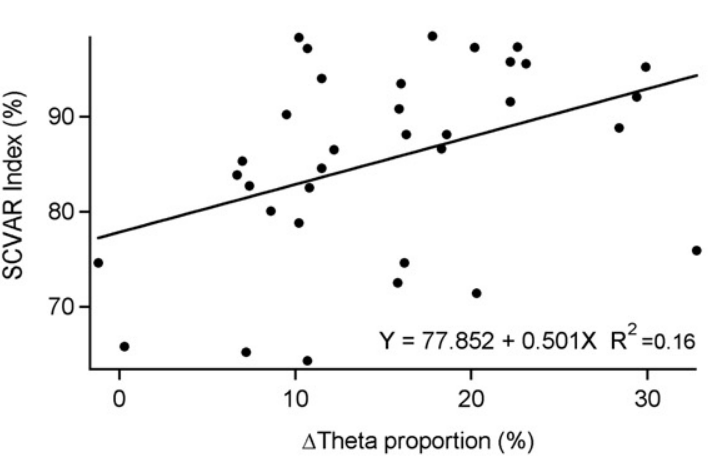

Fig. 3 - Relationship between hippocampal theta and SCVARs. (A) Left axis and $Z$ axis: hippocampal EEG power spectra (Fourier transformation of raw EEG signal, magnitude squared, $1.28 \mathrm{~s}$ epochs, $2.56 \mathrm{~s}$ bins), color coded (log scale) as in at top right. Right axis, red trace: percentage of EEG power in 5-8 Hz theta band in relation to total power (2.56 s bins). Right, black trace: mean tail artery blood flow. Example of the relation between changes evoked by a stimulus (drop) in tail artery blood flow and hippocampal EEG theta. (B) Group data (mean \pm SEM) for percentage of EEG power in 5-8 $\mathrm{Hz}$ theta band in relation to total power $(2.56 \mathrm{~s}$ bins) before and after stimuli. *Increases in theta proportion in relation to values pre-stimuli, $\mathrm{p}<0.05$. (C) Linear regression of $\Delta$ theta proportion (\%) and SCVAR index (\%), showing that theta proportion had a significant effect on the SCVAR index magnitude $\left(F_{(1,33)}=6.2, p=0.02, r^{2}=0.16, n=34\right.$ stimuli).

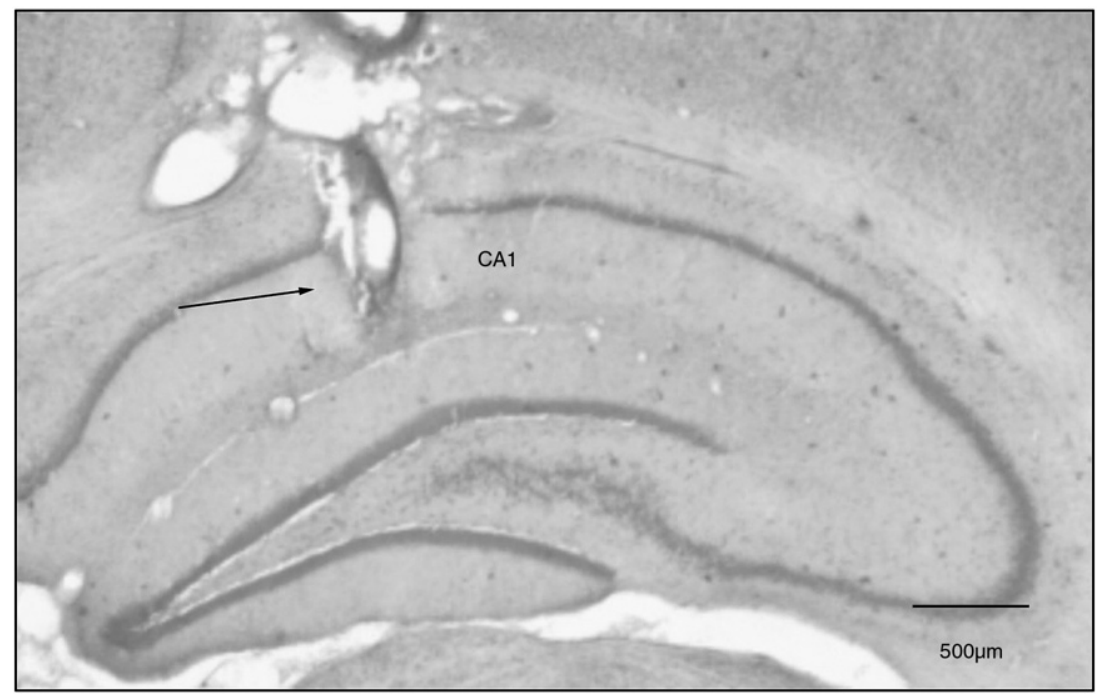

Fig. 4 - Location of electrode in the hippocampus. Neutral red stain of a coronal brain section showing a typical location of an EEG electrode in the hippocampal CA1 area (arrow). 
the other hand, activation of these neurons, in anaesthetized rats, results in vasoconstriction of the tail artery (Blessing and Nalivaiko, 2001).

We also confirmed the findings of previous investigations in rats demonstrating that salient environmental stimuli (i.e. stimuli that alert the animal) induce the increase of theta power in the hippocampal EEG (Montoya et al., 1989; Sainsbury et al., 1987). There are two distinct types of hippocampal theta rhythm in behaving animals. Theta that occurs concurrently with motor behaviors such as exploration and jumping is denominated type 1 theta (Bland, 1986; Vanderwolf, 1969). On the other hand, type 2 theta only appears when the animals are immobile and is induced by environmental stimuli (Montoya et al., 1989; Sainsbury et al., 1987). Several regions in the brain, including the amygdala, the posterior hypothalamus, and the median raphe, are able to influence theta to some degree. The medial septum (MS) is considered to be the main regulator of the hippocampal theta rhythm, because lesions of this region abolish theta (Oddie and Bland, 1998; Sainsbury and Bland, 1981; Winson, 1978).

We noted, in agreement with previous findings, that detection of theta activity after stimuli is more complex in rats than in rabbits. Rats move around more often than rabbits, so that type 1 (movement related) hippocampal theta is much more prominent, and background EEG patterns complicate the detection of type 2 (salient stimuli related) theta rhythm (Bland, 1986; Vanderwolf, 1969). To control for this effect, we continuously observed the animal via a video camera so that we could administer the alerting stimuli when the animal was still, with minimal type 1 theta. Despite the fact that we tried to make sure that the animals were immobile prior to the stimulus, eight stimuli had to be excluded from the analysis because the animals were exploring, thus, causing appearance of type 1 theta.

We demonstrated that the tail artery commences vasoconstriction on approximately $1 \mathrm{~s}$ after the onset of the increase in the proportion of theta power in the hippocampus. Since the conduction velocity of sympathetic neurons/axons can vary between $0.6 \mathrm{~m} / \mathrm{s}$ and $3.4 \mathrm{~m} / \mathrm{s}$ (depending on the level of the transmission) (Barman et al., 1995; Habler et al., 1999; Morrison et al., 1988; Vanegas et al., 1984), the conduction time between the brain and the tail artery smooth muscle is probably around $400 \mathrm{~ms}$, including synapses. After the electrical stimulus arrives in the neuromuscular junction the smooth muscle takes an additional $300 \mathrm{~ms}$ to start the contraction (Bao, 1993). The additional 300 ms gap between the increase in the proportion of theta power and tail artery vasoconstriction could possibly be due to cognition processing involved in deciding if the stimulus is a potential threat. The expression of the SCVAR over the next few seconds correlates with the proportion of theta power relative to total power. The increase in hippocampal theta power proportion before the SCVAR occurrence reinforces the idea that SCVARs are part of the alert response, as proposed previously (Yu and Blessing, 1997b). In our experiments, the stimuli that resulted in lowmagnitude SCVARs also resulted in small increases in hippocampal theta proportion suggesting that the SCVARs magnitude would depend on how much significance the animal assign to a given stimulus. Accordingly, Vertes (2005) hypothesized that theta would serve as a "significance signal" to the hippocampus, which means that the information arriving in the hippocampus while theta is present is more likely to be stored than the information that arrives without theta (Vertes, 2005).

Although the commencing of the two responses (i.e. hippocampal theta and SCVARs) is correlated, which shows an integration of the responses, the time courses of these responses are not. Typically, hippocampal theta rhythm proportion values return to the pre-stimulus values within $25 \mathrm{~s}$ after the stimulus. On the other hand, the blood flow to the tail artery did not return to baseline for, at least, 1 minute after the stimulus. The time courses of the SCVARs response were particularly inconsistent varying from 1 minute to 6 minutes depending on the animal and on the stimulus type (data not shown). The lack of correlation in the time course of these two variables is not unexpected. We believe that the appearance of theta indicates that the animal detected the stimulus and regarded that particular stimulus as a possible threat. As a result, the circuitry involved in generating SCVARs (i.e. vasoconstriction of the tail artery) would then be activated. The duration of the SCVARs would not depend on the duration of hippocampal theta given that the stimulus was already detected and "classified" as a potential danger. From there on, other areas, such as the amygdala, would be responsible to determine if the danger was over and to terminate the response when appropriate.

The most common behavior observed during the period where proportion of theta was increased was freezing accompanied by lateral head movements. This behavior is typically attributed to risk assessment behaviors (Blanchard and Blanchard, 1988). Risk assessment is an essential factor to establish the quality and the nature of the potential threat (Blanchard and Blanchard, 1988, 1989; Blanchard et al., 2001). Thus, the increase in theta proportion observed in our experiments could be part of the cognitive process that takes place during risk assessment behaviors, which could facilitate the characterization of a potential threat.

The amygdala is a possible candidate to be coordinating both the occurrence of increased hippocampal theta rhythm and the SCVARs due to detection of stimuli. Amygdalahippocampal interactions are involved in the conversion of short-term memory into long-term fear memory related to emotional arousal (McGaugh et al., 1996; McGaugh, 2004; Richter-Levin, 2004). Yu and Blessing $(1999,2001)$ have shown that inactivation of the amygdala in rabbits prevents SCVARs produced by various non-noxious stimuli. We propose that when the amygdala identifies a stimulus as relevant, hippocampal theta rhythm would be modulated by this nucleus. In addition, the amygdala could activate downstream pathways, possibly by activating pre-sympathetic neurons located in the medulla (Nalivaiko and Blessing, 2001), to produce SCVARs. For instance, inactivation of medullary raphe neurons in rats prevented falls in cutaneous blood flow that normally occur in response to alerting stimuli (Ootsuka and Blessing, 2005). On the other hand, activation of these neurons, in anaesthetized rats, results in vasoconstriction of the tail artery (Blessing and Nalivaiko, 2001). Consequently, SCVAR occurrence after a stimulus would depend on the amygdala determining an event as being a relevant one. This process would be part of the assess- 
ment behavior that usually occurs during situations of potential danger.

However, more studies have to be done to determine the areas of the brain that could be involved in coordinating the interaction between SCVAR and hippocampal theta rhythm.

\section{Experimental procedures}

\subsection{Animals}

All experiments were performed in male Sprague-Dawley rats weighing $300 \pm 20 \mathrm{~g}(n=7)$. Animals were initially housed in groups and maintained in a 12 hour/12 hour light-dark cycle, lights on at $0700 \mathrm{~h}$. Animals had free access to water and food. Experiments were performed in accordance with the European Community Council Directive of November 24, 1986 (86/ $609 /$ EEC), and were approved by the Animal Welfare Committee of Flinders University. Animals were instrumented under general anaesthesia (isoflurane in $\mathrm{O}_{2}$; Veterinary Companies of Australia Pty. Ltd., NSW, Australia). At the end of surgical procedures, analgesic (Caprofen, $5 \mathrm{mg} / \mathrm{kg} / \mathrm{ml} \mathrm{s.c.)} \mathrm{and} \mathrm{anti-}$ biotics (Baytril, $15 \mathrm{mg} / \mathrm{kg}$ s.c.) were administered. All animals were returned to the animal house in individual cages. Animals recovered for 7 days before any experimental procedures.

\subsection{Surgical procedures}

Doppler ultrasonic flow probes (Iowa Doppler Products, IA, USA) were employed to measure tail and superior mesenteric artery blood flow. The tail flow probe implantation procedure was performed according the technique developed in our laboratory (Blessing, 2005; Blessing and Ootsuka, 2007; Garcia et al., 2001). A second Doppler blood flow probe was positioned in the superior mesenteric artery. A $4 \mathrm{~cm}$ midline incision was made in the abdomen. The intestines were put aside with gauze moistened in warm Ringer's solution. The mesenteric artery was isolated from the mesenteric lymph duct, and the blood flow probe (1.3 $\mathrm{mm}$, internal diameter) was positioned around the artery origin. Surgical glue (Loctite Pty. Ltd., NSW, Australia) was applied to the base of the probe to keep the probe in place. Wires from the probe were brought out through the abdominal wall and passed subcutaneously to the back of the neck. The incision was sutured and an analgesic (bupivacaine, $5 \mathrm{mg} / \mathrm{kg}$ i.m.) was injected around the incision.

Animals were then positioned in a stereotaxic apparatus with the incisor bar positioned at $3.3 \mathrm{~mm}$ below the level of the interaural line. A $2 \mathrm{~cm}$ incision was made on the top of head, and the skull was cleaned of all connective tissue. Skull burr roles were made on the parietal bones, and two stainless steel screws were positioned for anchoring. Reference and ground electrodes were screwed to the frontal and occipital bones, respectively. Two stainless steel guide cannulae (23G) were positioned directly above the dorsal hippocampus, $3.8-4.0 \mathrm{~mm}$ caudal to bregma, 1.9-2.1 mm from midline, and 1.8-2.0 mm ventral from the skull (Paxinos and Watson 2007). A Tefloninsulated silver wire (0.0110 inches, coated) was inserted through the guide cannulae and its tip positioned $1 \mathrm{~mm}$ below the tip of the guide cannula, in CA1 area. EEG electrode and blood flow probe wires were connected to a head socket via Teflon-insulated stainless steel wires and fixed in place with dental cement.

Tail artery blood flow signals were converted to voltage outputs (Triton Technology, San Diego, CA, USA) and digitalized, with a sample rate of $40 \mathrm{~Hz}$, using a MacLab analog-todigital converter (AD Instruments, Castle Hill, NSW, Australia). EEG signals were acquired through voltage buffer preamplifiers situated in the headpiece that was located at the end of a flexible cable. The cable was connected to a swivel device to allow animals to move freely. Signals were further preamplified with a MacLab bridge amplifier unit (0.1-100 band-pass), subsequently digitalized with MacLab, and transferred to an Apple computer and displayed using Chart software (AD Instruments, Castle Hill, NSW, Australia).

\subsection{Experimental design}

On the day of experiments, the rats were brought to the experimental room at least 2 hours before the commencement of the experiments. A temperature-controlled, lightproof box was used as experimental cage in all experiments. Animals were transferred from their home cage to the box and connected to the swivel device 1 hour before the experiments commenced. Cage temperature was maintained at $24-28^{\circ} \mathrm{C}$. A Web camera (Sonix, Taiwan) was positioned on the top of the cage to monitor the animal's activity during the entire experiment.

Animals were left undisturbed for at least 1 hour prior to the beginning of the experiments. The rats were then subjected to a series of 6 standardized stimuli (Blessing, 2005; Blessing and Ootsuka, 2007) administered in the same order in all rats. Since movement can induce theta rhythm appearance, we used the camera to monitor the rat and make sure that the stimuli were only given when the animal was immobile. A flexible metal rod, released from a restraint, tapped the side of the cage. A sound $(0.5 \mathrm{~s}, 90 \mathrm{~dB}, 1000 \mathrm{~Hz})$ was displayed right outside the box. The cage was suddenly dropped $1 \mathrm{~cm}$ by removal of a support. The cage was vigorously moved from side to side. A window of $15 \times 15 \mathrm{~cm}$, located in the front part of the box, was opened. Finally, the door was opened, and the rat was pricked with a $23 \mathrm{G}$ needle. A vibration detector device located on the side of the cage and connected to MacLab detected the timing of each stimulus.

\subsection{Histology}

After completion of experiments, rats were deeply anaesthetized with pentobarbital $(100 \mathrm{mg} / \mathrm{kg})$, and a current $(150 \mu \mathrm{V})$ was passed for $30 \mathrm{~s}$ through the electrodes to produce lesions in the hippocampus to mark the electrode position. Rats were then perfused transcardially with $100 \mathrm{ml}$ of saline followed by $120 \mathrm{ml}$ of phosphate buffer containing $10 \%$ formaldehyde and $4 \%$ glutaraldehyde. The brain was then removed, stored in the formaldehyde and glutaraldehyde solution overnight, then transferred to a $20 \%$ sucrose solution until saturation. Coronal brain sections $(40 \mu \mathrm{m})$ at the level of the hippocampus were cut on a cryostat and mounted on gelatinized slides. After 24 hours, the sections were stained with neutral red (5\%) for analysis of electrode position. 


\subsection{Data analysis}

For the analysis of the change in blood flow after stimuli, we calculated the mean blood flow and the mean pulse amplitude for a period of $3 \mathrm{~s}$ immediately before the stimulus and for a period of $3 \mathrm{~s}$ from a period approximately $10 \mathrm{~s}$ after the administration of each stimulus. The SCVAR index was calculated as a percentage fall from pre-alerting blood flow values using both mean blood flow and mean pulse amplitude. The formula used to calculate the SCVAR index was as follows: 100 -[(baseline mean flow+baseline mean pulse amplitude)/ (mean flow after stimulus + mean pulse amplitude after stimulus) $\times 100$ (Blessing, 2005).

The SCVAR index was calculated in all rats for each individual stimulus for both tail flow and mesenteric flow. An overall SCVAR index, for both flows, was achieved by averaging the SCVAR index of each stimulus in all rats. Data from stimuli trials in which the animal was exploring at the time of the stimulus were not included in the analysis. This resulted in the exclusion of a total of eight (8) stimuli trails (i.e. from different animals).

For quantification of theta rhythm, the hippocampal EEG record was divided in $2.56 \mathrm{~s}$ windows. Each window was divided in 64 point epochs. Both ends of the epochs were smoothed with Hamming window, and the magnitude of the Fourier transform, for each epoch, was obtained using IgorPro software (WaveMetrics, Lake Oswega, OR, USA). The relative magnitude of each frequency was averaged for each window and then displayed graphically. A band-pass filter was used with the filter set up at 1-20 Hz. The proportion of theta was evaluated as a proportion of the total area under frequencymagnitude area obtained between 5 and $8 \mathrm{~Hz}$.

\subsection{Statistical analysis}

Data were analysed with IgorPro and Statview (SAS Institute, Cary, NC, USA) software. Pre- and post-stimuli values of hippocampal theta proportion were compared using repeatedmeasures analysis of variance (ANOVA). Fisher's protected post-hoc test was used to determine statistical significance differences. Linear regression (Statview) was used to quantify the relationship between increases in hippocampal theta proportion and SCVAR index. SCVAR index values of tail and mesenteric blood flow were compared using a paired Student t-test. The significance threshold level was set at 0.05 .

\section{Acknowledgments}

We thank Robyn Flook, Sarah Todd, and Mal Lomu for technical assistance. This work was supported by grants 426713 and 426716 from the NHMRC of Australia and by the Flinders Medical Centre Foundation.

\section{REFEREN C ES}

Bao, J.X., 1993. Sympathetic neuromuscular transmission in rat tail artery: a study based on electrochemical, electrophysiological and mechanical recording. Acta. Physiol. Scand. Suppl. 610, 1-58.
Barman, S.M., Orer, H.S., Gebber, G.L., 1995. Axonal projections of caudal ventrolateral medullary and medullary raphe neurons with activity correlated to the $10-\mathrm{Hz}$ rhythm in sympathetic nerve discharge. J. Neurophysiol. 74, 2295-2308.

Berry, S.D., Seager, M.A., 2001. Hippocampal theta oscillations and classical conditioning. Neurobiol. Learn. Mem. 76, 298-313.

Blanchard, D.C., Blanchard, R.J., 1988. Ethoexperimental approaches to the biology of emotion. Annu. Rev. Psychol. 39, 43-68.

Blanchard, R.J., Blanchard, D.C., 1989. Antipredator defensive behaviors in a visible burrow system. J. Comp. Psychol. 103, 70-82.

Blanchard, R.J., Yang, M., Li, C.I., Gervacio, A., Blanchard, D.C., 2001. Cue and context conditioning of defensive behaviors to cat odor stimuli. Neurosci. Biobehav. Rev. 25, 587-595.

Bland, B.H., 1986. The physiology and pharmacology of hippocampal formation theta rhythms. Prog. Neurobiol. 26, 1-54.

Bland, B.H., Sainsbury, R.S., Seto, M., Sinclair, B.R., Whishaw, I.Q., 1981. The use of sodium pentobarbital for the study of immobility-related (type 2) hippocampal theta. Physiol. Behav. 27, 363-368.

Blessing, W.W., 2005. Clozapine increases cutaneous blood flow and reduces sympathetic cutaneous vasomotor alerting responses (SCVARs) in rats: comparison with effects of haloperidol. Psychopharmacology 181, 518-528.

Blessing, W.W., Nalivaiko, E., 2001. Raphe magnus/pallidus neurons regulate tail but not mesenteric arterial blood flow in rats. Neuroscience 105, 923-929.

Blessing, W.W., Ootsuka, Y., 2007. Activation of dopamine $\mathrm{D}_{2}$ receptors in the CNS inhibits sympathetic cutaneous vasomotor alerting responses (SCVARs), contributing to clozapine's SCVAR-inhibiting action. Prog. Neuro-Psychopharmacol. Biol. Psychiatry 31, 328-336.

Garcia, J.N., Pedersen, N.P., Nalivaiko, E., Blessing, W.W., 2001. Tail artery blood flow measured by chronically implanted Doppler ultrasonic probes in unrestrained conscious rats. J. Neurosci. Methods 104, 209-213.

Habler, H., Bartsch, T., Janig, W., 1999. Rhythmicity in single fiber postganglionic activity supplying the rat tail. J. Neurophysiol. 81, 2026-2036.

Kistler, A., Mariauzouls, C., von Berlepsch, K., 1998. Fingertip temperature as an indicator for sympathetic responses. Int. J. Psychophysiol. 29, 35-41.

McGaugh, J.L., 2004. Memory reconsolidation hypothesis revived but restrained: theoretical comment on Biedenkapp and Rudy (2004). Behav. Neurosci. 118, 1140-1142.

McGaugh, J.L., Cahill, L., Roozendaal, B., 1996. Involvement of the amygdala in memory storage: interaction with other brain systems. Proc. Natl. Acad. Sci. U. S. A. 93, 13508-13514.

Montoya, C.P., Heynen, A.J., Faris, P.D., Sainsbury, R.S., 1989. Modality specific type 2 theta production in the immobile rat. Behav. Neurosci. 103, 106-111.

Morrison, S.F., Milner, T.A., Reis, D.J., 1988. Reticulospinal vasomotor neurons of the rat rostral ventrolateral medulla: relationship to sympathetic nerve activity and the C1 adrenergic cell group. J. Neurosci. 8, 1286-1301.

Nalivaiko, E., Blessing, W.W., 2001. Raphe region mediates changes in cutaneous vascular tone elicited by stimulation of amygdala and hypothalamus in rabbits. Brain Res. 891, 130-137.

Oddie, S.D., Bland, B.H., 1998. Hippocampal formation theta activity and movement selection. Neurosci. Biobehav. Rev. 22, 221-231.

Ootsuka, Y., Blessing, W.W., 2005. Inhibition of medullary raphe/parapyramidal neurons prevents cutaneous vasoconstriction elicited by alerting stimuli and by cold exposure in conscious rabbits. Brain Res. 1051, 189-193. 
Paxinos, G, Watson, C, 2007. The Rat Brain in Stereotaxic Coordinates. Elsevier Academic Press., San Diego. Vol. 6.

Richter-Levin, G., 2004. The amygdala, the hippocampus, and emotional modulation of memory. Neuroscientist 10, 31-39.

Sainsbury, R.S., Bland, B.H., 1981. The effects of selective septal lesions on theta production in CA1 and the dentate gyrus of the hippocampus. Physiol. Behav. 26, 1097-1101.

Sainsbury, R.S., Montoya, C.P., 1984. The relationship between type 2 theta and behavior. Physiol. Behav. 33, 621-626.

Sainsbury, R.S., Heynen, A., Montoya, C.P., 1987. Behavioral correlates of hippocampal type 2 theta in the rat. Physiol. Behav. 39, 513-519.

Sokolov, E.N., 1963. Higher nervous functions; the orienting reflex. Annu. Rev. Physiol. 25, 545-580.

Vanderwolf, C.H., 1969. Hippocampal electrical activity and voluntary movement in the rat. Electroen. Clin. Neuro. 26, 407-418.

Vanegas, H., Barbaro, N.M., Fields, H.L., 1984. Tail-flick related activity in medullospinal neurons. Brain Res. 321, 135-141.
Vertes, R.P., 2005. Hippocampal theta rhythm: a tag for short-term memory. Hippocampus 15, 923-935.

Vertes, R.P., Kocsis, B., 1997. Brainstem-diencephaloseptohippocampal systems controlling the theta rhythm of the hippocampus. Neuroscience 81, 893-926.

Winson, J., 1978. Loss of hippocampal theta rhythm results in spatial memory deficit in the rat. Science 201, 160-163.

Yu, Y.H., Blessing, W.W., 1997a. Acute increases in forebrain blood flow during altering responses in conscious rabbits. Brain Res. 767, 1-7.

Yu, Y.H., Blessing, W.W., 1997b. Cutaneous vasoconstriction in conscious rabbits during alerting responses detected by hippocampal theta-rhythm. Am. J. Physiol. 272, R208-R216.

Yu, Y.H., Blessing, W.W., 1999. Amygdala co-ordinates sudden falls in ear pinna blood flow in response to unconditioned salient stimuli in conscious rabbits. Neuroscience 93, 135-141.

Yu, Y.H., Blessing, W.W., 2001. Neurons in amygdala mediate ear pinna vasoconstriction elicited by unconditioned salient stimuli in conscious rabbits. Auton. Neurosci. 87, 236-242. 